\title{
MHD Convection Fluid Flow and Heat Transfer in an Inclined Microchannel with Heat Generation
}

\author{
Hasan Nihal Zaidi ${ }^{1}$, Naseem Ahmad ${ }^{2}$ \\ ${ }^{1}$ Department of Basic Sciences, College of Preparatory Year, University of Hail, Hail, Kingdom of Saudi Arabia \\ ${ }^{2}$ Department of Mathematics, Jamia Millia Islamia, New Delhi, India
}

Email address:

hasannihalzaidi@hotmail.com (H. N.Zaidi),Naseem_mt@yahoo.com (N. Ahmad)

To cite this article:

Hasan Nihal Zaidi, Naseem Ahmad. MHD Convection Fluid Flow and Heat Transfer in an Inclined Microchannel with Heat Generation. American Journal of Applied Mathematics. Vol. 5, No. 5, 2017, pp. 124-131. doi: 10.11648/j.ajam.20170505.11

Received: April 29, 2017; Accepted: August 9, 2017; Published: September 22, 2017

\begin{abstract}
The problem of fully developed free convection flow of electrically conducting fluid in an inclined microchannel in the presence of transverse magnetic field and internal heat generation is investigated. The analytical solution for velocity profile and temperature profile have been obtained, considering the velocity slip and temperature jump conditions at the wall of the microchannel. The effect of different parameters involved in the problem on the velocity and temperature profile along with the skin friction parameter and Nusselt number has been discussed graphically.
\end{abstract}

Keywords: Magnetohydrodynamic (MHD), Free Convection, Heat Generation, Microchannel, Velocity Slip, Temperature Jump

\section{Introduction}

The study of micro-electro-mechanical system (MEMS) and nano-electrical-mechanical systems (NEMS) has attracted much attention to design micro-devices such as micro-motors, micro-sensors, micro-mechanical gyroscopes, micro-pumps, micro valves, micro-rockets, micro-gasturbines, micro-heat-exchangers, biological and chemical devices etc. Microchannels are used to transport biological material such as protein, DNA, cells and embryos or to transport chemical samples and analytes. Advantage of microchannels is due to their high surface to volume ratio and their small volume. The large surface to volume ratio increases the rate of heat and mass transfer that makes micro devices excellent tools. Flow in heat transfer and chemical reactor devices are usually faster than, those in biological devices and chemical analysis microdevices. These applications have motivated scholars to understand the flow behaviors in these small systems to enhance the performance during the design process.

Fluid flow through micro channel is modeled using either the continuum or the molecular approach depending on the Knudsen number, $K_{n}$. It is defined as the ratio between the fluid's mean free path to the channel gap $(K n=\lambda / b)$. The magnitude of the Knudsen number determines the appropriate gas dynamic regime. If the Knudsen number, is greater than $10^{-3}[2,3]$, nonequilibrium effect may start to occur. Modified slip boundary condition can be used in continuum models for Knudsen numbers between $10^{-1}$ and $10^{-3}$ [3]. The continuum assumptions and fluid theory are not applicable when the Knudsen number continues to increase.

In recent years several investigations have been conducted in the microchannels and microtubes. Arkilic et al [1] have been studied the gaseous slip flow in a long microchannels. Later Chen and Weng [4] investigated the fully developed natural convection in an open ended vertical parallel plate microchannel with asymmetric wall temperature distribution. Weng and Chen $[5,6]$ studied the role of variable physical properties and thermal creep in fully developed convection flow at microscale. The transient hydrodynamics and thermal behaviors of fluid flow in an open ended vertical parallel plate microchannel under the hyperbolic heat conduction model, were investigated by A. F. Khadrawi et al [14]. Natural convection slip flow in a vertical microchannel heated at uniform heat flux had been studied by B. Buonomo and $\mathrm{O}$. Manca [7].

Magnetohydrodynamic (MHD) flow and heat transfer of 
electrically conducting and heat generating fluids through channel or pipe flow attracted many scholars due to its used in many applications such as Magnetohydrodynamic (MHD) generators, pumps, accelerators and flow meter. The MHD channel flow was first investigated by Hartmann [17]. Osterle and Young [19] later investigated the natural convection between heated vertical plates with magnetic field. Seigal [18], Perlmutter and Siegel [20], Romig [21], Affiliated with Department of Mathematics, Indian Institute of Technology Chamkha [11], Umavathi [22] and Naseem Ahmad et al [23]. have presented detailed analysis of free and forced convection heat transfer to in the presence of magnetic field for the vertical channel. Recently Chamkha et al. $[12,13]$ studied the MHD free convection flow of a nanofluid past a vertical Plate in the presence of heat generation or absorption effects. B. K. Jha et al [10], investigated the MHD natural convection in a vertical parallel plate microchannel considering velocity slip and temperature jump conditions at the fluid- wall interface. Hasan Nihal Zaidi and Naseem Ahmad had been discussed MHD convection flow of two immiscible fluids in an inclined channel with heat generation or absorption [15]. Chi Chuan Wang et al [16] experimentally investigated the effect of inclination on the convective boiling performance of a microchannel heat sink. They found that the heat transfer coefficient for $45^{\circ}$ upward considerably exceeds other configurations.

In this paper, we discussed an analytic study of steady MHD free convective flow of an incompressible electrically conducting fluid through an inclined microchannel taking into account heat generation and influence of a uniform magnetic field $B_{0}$.

\section{Mathematical Formulation}

Considering fully developed laminar flow of electrically conducting incompressible viscous fluid through an inclined microchannel formed by two parallel plates extending in the $x$ and $z$ directions, making an angle $\alpha$ with the horizontal. The plates of the channel are placed at a distance $h$ apart and maintained at different temperatures $T_{1}$ and $T_{2}\left(T_{2}>T_{1}\right)$. A constant magnetic field of strength $B_{0}$ is applied transverse to the flow field.

The governing equation of motion and energy in the dimensionless form in the presence of velocity slip and temperature jump under Boussinesq's approximation are:

$$
\begin{gathered}
\frac{d^{2} u}{d y^{2}}-M^{2} u+G r \sin \alpha \theta=0 \\
\frac{d^{2} \theta}{d y^{2}}+\varphi \cdot \theta=0
\end{gathered}
$$

Here, in the equations (1) and (2), the magnetic Reynolds number is assumed to be small so that the induced magnetic field is neglected. In addition the viscous dissipation and material derivative of the pressure are also be neglected.

Introducing the following dimensionless quantities:

$$
\begin{gathered}
y^{*}=\frac{y}{h}, \theta=\frac{T-T_{0}}{T_{1}-T_{0}}, u^{*}=\frac{u}{u_{0}}, M^{2}=\frac{\sigma B_{0}^{2} h^{2}}{\mu}, \phi=\frac{Q h^{2}}{k}, \operatorname{Pr}=\frac{\mu c_{p}}{k}, \\
G r=\frac{g \beta\left(T_{1}-T_{0}\right) h^{2}}{v u_{0}}
\end{gathered}
$$

The dimensionless boundary conditions for velocity slip and temperature jump are:

$$
u(0)=\beta_{v} K n\left(\frac{d u}{d y}\right)_{y=0}, u(1)=-\beta_{v} K n\left(\frac{d u}{d y}\right)_{y=1}
$$

where: $\beta_{v}=\frac{2-\sigma_{v}}{\sigma_{v}}, \beta_{t}=\left(\frac{2-\sigma_{t}}{\sigma_{t}}\right) \cdot\left(\frac{2 \gamma_{s}}{\gamma_{s}+1}\right) \cdot \frac{1}{P r}, K n=\frac{\lambda}{h}, \ln =\frac{\beta_{t}}{\beta_{v}}, \eta=\frac{T_{2}-T_{0}}{T_{1}-T_{0}}$

Here $K n, \gamma_{s}, \sigma_{v}, \sigma_{t}, \beta_{s}, \beta_{t}, \operatorname{Pr}$ and $\ln$ are Knudsen number, the ratio of specific heats, tangential momentum coefficient, thermal accommodation coefficient, Prandtl number and fluid wall interaction parameter, respectively.

The solutions of equations (1) and (2) for the heat generating fluids $(+\phi>0)$ is obtained by using the conditions (4) and (5) are:

$$
\begin{gathered}
\theta=c_{1} \cos (\sqrt{\varphi} y)+c_{2} \sin (\sqrt{\varphi} y) \\
u=c_{3} \mathrm{e}^{\mathrm{My}}+c_{4} \mathrm{e}^{-\mathrm{My}}+a_{2}\left\{c_{1} \cos (\sqrt{\varphi} y)+c_{2} \sin (\sqrt{\varphi} y)\right\}
\end{gathered}
$$

The Rate of heat transfer over the microchannel walls is described by the Nusselt number, define on the both walls as:

$$
\begin{gathered}
N u_{1}=\frac{q h}{\left(T_{1}-T_{0}\right) k}=\left.\frac{d \theta}{d y}\right|_{y=0}=c_{2} \sqrt{\phi} \\
N u_{2}=\frac{q h}{\left(T_{1}-T_{0}\right) k}=\left.\frac{d \theta}{d y}\right|_{y=1}=\sqrt{\phi} \cdot\left[-c_{1} \sin (\sqrt{\phi})+c_{2} \cos (\sqrt{\phi})\right]
\end{gathered}
$$

Once the velocity distribution is known, the skin friction $(\tau)$ on the walls of microchannel can be calculated as follows:

$$
\tau_{1}=\left.\frac{d u}{d y}\right|_{y=0}=M \cdot c_{3}-M \cdot c_{4}+a_{2} c_{2} \sqrt{\phi}
$$




$$
\tau_{2}=\left.\frac{d u}{d y}\right|_{y=1}=M c_{3} \mathrm{e}^{\mathrm{M}}-M c_{4} \mathrm{e}^{-\mathrm{M}}+a_{2} \sqrt{\phi}\left\{-c_{1} \sin (\sqrt{\phi})+c_{2} \cos (\sqrt{\phi})\right\}
$$

where,

$$
\begin{gathered}
c_{1}=\frac{a_{1}+\eta a_{4}}{a_{4}-a_{1} a_{3}}, c_{2}=\frac{1-\eta a_{3}}{a_{4}-a_{1} a_{3}}, c_{3}=\frac{a_{5} a_{7} e^{-M}-a_{6} a_{8}}{a_{9}}, c_{4}=\frac{a_{5} a_{8}-a_{6} a_{7} e^{M}}{a_{9}} \\
a_{0}=\beta_{v} K n \sqrt{\phi}, a_{1}=\beta_{v} K n \ln \sqrt{\phi}, a_{2}=\frac{G r \sin \alpha}{\phi+M^{2}} \\
a_{3}=\cos (\sqrt{\phi})-a_{1} \sin (\sqrt{\phi}), a_{4}=\sin (\sqrt{\phi})+a_{1} \cos (\sqrt{\phi}) \\
a_{5}=1-M \beta_{v} K n, a_{6}=1+M \beta_{v} K n \\
a_{7}=a_{2}\left(a_{0} c_{2}-c_{1}\right), a_{8}=a_{2}\left[c_{1}\left\{a_{0} \sin (\sqrt{\phi})-\cos (\sqrt{\phi})\right\}-c_{2}\left\{a_{0} \cos (\sqrt{\phi})+\sin (\sqrt{\phi})\right\}\right] \\
a_{9}=a_{5}^{2} e^{-M}-a_{6}^{2} e^{M}
\end{gathered}
$$

\section{Result and Discussion}

The analytical solution for the problem of natural convection in an inclined microchannel can be obtained in the presence of transverse magnetic field. The effects of different parameters on the velocity profile, temperature profile, rate of heat transfer and skin friction are discussed with the aid of the graphs. The present analysis is performed over the ranges $0 \leq K n \leq 0.1,0 \leq \ln \leq 3$ and $1 \leq M \leq 2$. For simplicity, the values $\beta_{v}$ and $\beta_{t}$ are assumed equal to 1.0. The values of $K n$ and $l n$ for the analysis are 0.05 and 1.667 respectively as presented by Chen and Weng [4].

Figure 1 is the graph of velocity profile for different values of $\eta$ and Kundsen number (Kn). It is observed that as the value of $K n$ increases, the slip velocity at the walls increases which reduces the retarding effect of the wall. This produces an increase in the gas velocity near the wall. It is also clear from the graph that as the temperature difference ratio $\eta$ increases the effect of $K n$ on the velocity profile becomes significant.

The effect of the temperature difference ratio $(\eta)$ and Hartmann number $(\mathrm{M})$ is shown in the figure 2. It is found that the increase in the Hartmann number suppresses the velocity field. This effect of Hartmann number on velocity profile becomes evident with the increase of $\eta$.

From figure 3 , it is found that the velocity slip increases on microchannel wall with the increase of fluid wall interaction parameter $(l n)$. Figure 4 shows the effect of the angle of inclination on the velocity profile, which depicts that velocity increases as the inclination angle increases. This is due to the increase of buoyancy effect with the inclination angle.

Figure 5 shows the variation of velocity profile for different values of heat generating coefficient $(\phi)$. It is clear from the graph that as the value of $\phi$ increases, the velocity of the fluid particles decreases at the hot plates. This has the tendency to decrease the buoyancy effects close to the hot plates and it produces a reduction in the fluid velocity.

Figure 6 displays the temperature distribution for various values of Knudsen number $(K n)$. It should be noted that as the value of $K n$ increases, the temperature increases.. It is also clear from the graph that as the temperature difference ratio $\eta$ increases, the effect of $K n$ on the temperature profile becomes significant.

Figure 7 depicts the temperature distribution for different values of fluid wall interaction parameter $(\ln )$. It is evident from figure 7 that the temperature increases with the increase of fluid wall interaction parameter $(\ln )$ and the temperature difference ratio $\eta$. Figure 8 shows the temperature profile for different values of heat generation coefficient $(\phi)$. It is clear from the graph that as the value of $\phi$ increases, the temperature increases.

The variation of rate of heat transfer is revealed in figure 9 and figure 10 with respect to fluid wall interaction parameter $(l n)$ and the Knudsen number $(K n)$ for different values of wall ambient temperature difference ratio $(\eta)$ at the walls of microchannel at $y=0$ and $y=1$, respectively. Figure 9 shows that as the value of $\ln$ increases the value of Nuselt number increases. Figure 10 shows that as the value of $l n$ increases, the value of Nusselt number decreases.

The skin friction variation for different values of fluid wall interaction parameter $(\ln )$ at the microchannel plate $y=0$ and $y=1$ is shown in the figures 11 and 12 respectively. It is evident from the graphs that as the value of fluid wall interaction parameter $(\ln )$ increases, the magnitude of skin friction increases. It is also found that the effect of fluid wall interaction parameter $(l n)$ is significant for $\eta=1$.

Figure 13 and 14 show the effect of Hartmann number $(M)$ for different values of wall ambient temperature difference ratio $(\eta)$ at the walls of microchannel $y=0$ and $y=1$, respectively. It is clear from the graphs that the magnitude of skin friction decreases with the increase of the value of Hartmann Number $(M)$. It is also evident from the graph that the magnitude of skin friction is higher in case of $\eta=1$.

\section{Conclusions}

The problem of steady, laminar flow of a viscous, incompressible, electrically conducting and heat generating fluid along an inclined microchannel in the presence of transverse magnetic field is solved analytically. A parametric study of the Knudsen number, Hartmann Number, Fluid wall 
interaction parameter, heat generation coefficient and angle of inclination is performed to illustrate their influence on the solutions. It is found that velocity of fluid particle increases with the increase of Knudsen number $(K n)$, fluid wall interaction parameter $(l n)$ and heat generating coefficient while it decreases with the increase of Hartmann number. In
Addition, it is observed that the rate of heat transfer increases at cooler plate but it decrease at hot plate with the increase of fluid wall interaction parameter. It is also interesting to note that magnitude of skin friction increases with the increase of fluid wall interaction parameter while it is decreases with the increase of Hartmann Number $(M)$.

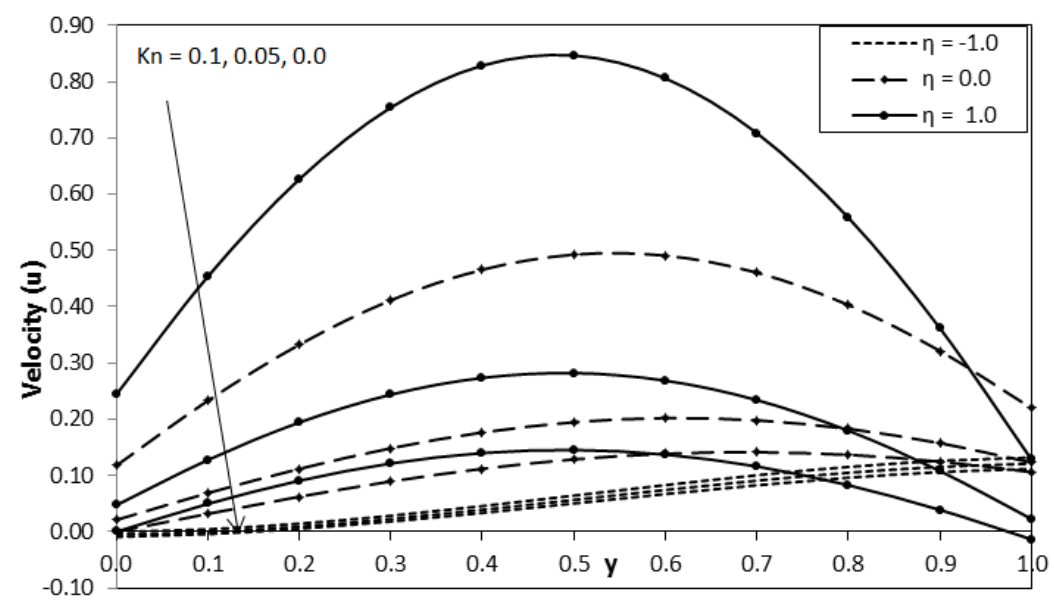

Figure 1. Effect of Kn and $\eta$ on velocity profile for $\ln =1.667, M=2.0, \alpha=45^{\circ}, \phi=5$.

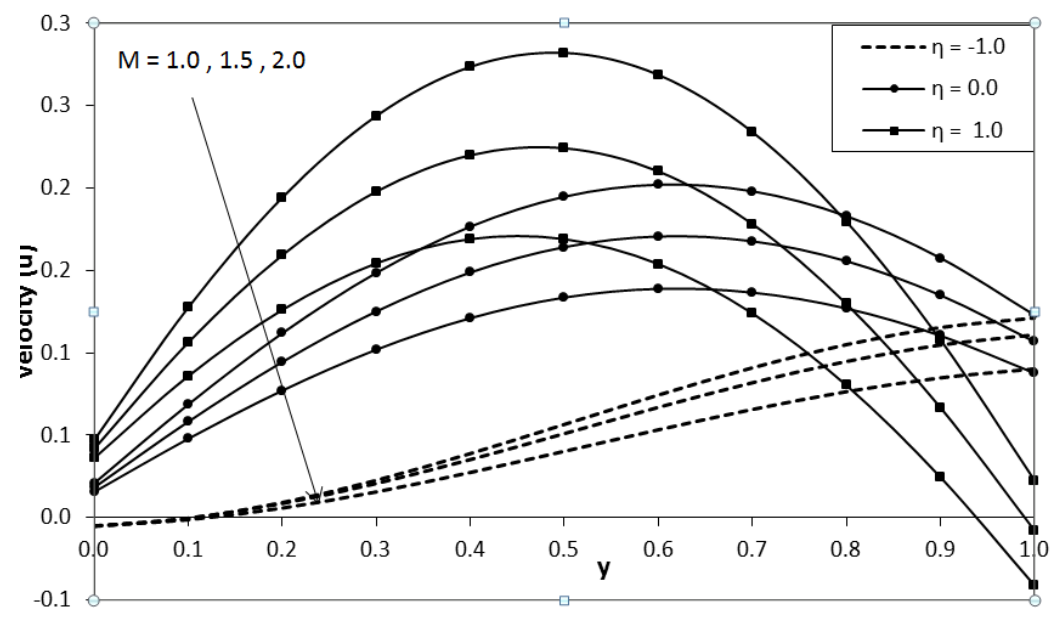

Figure 2. Effect of $M$ and $\eta$ on velocity profile for $\ln =1.667, K n=0.05, \alpha=45^{\circ}, \phi=5$.

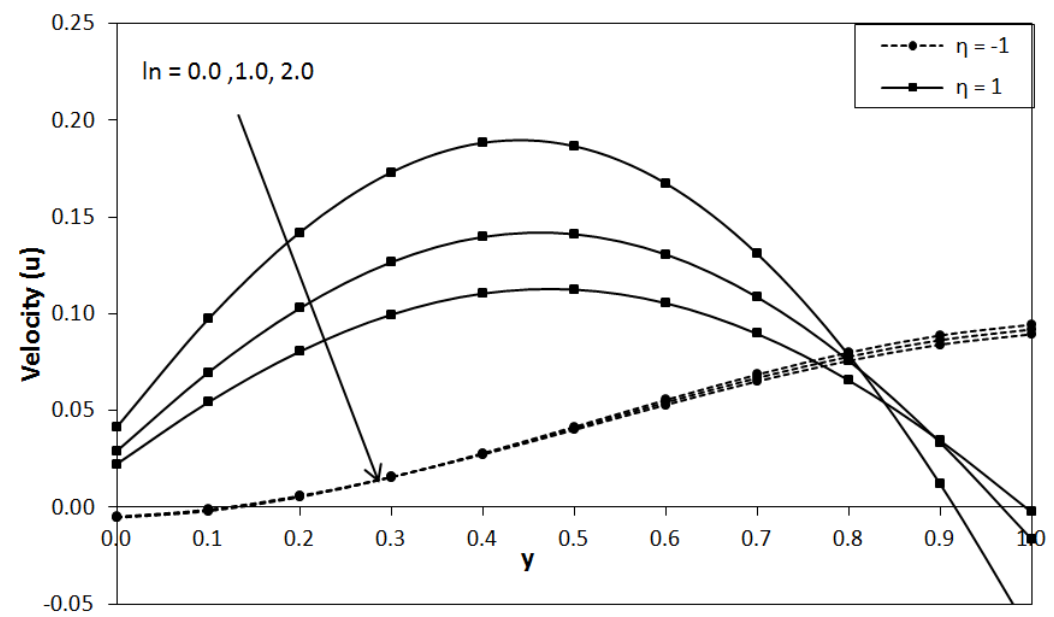

Figure 3. Effect of $\ln$ and $\eta$ on velocity profile for $=0.05, \ln =1.667, \alpha=45^{0}, \phi=5$. 


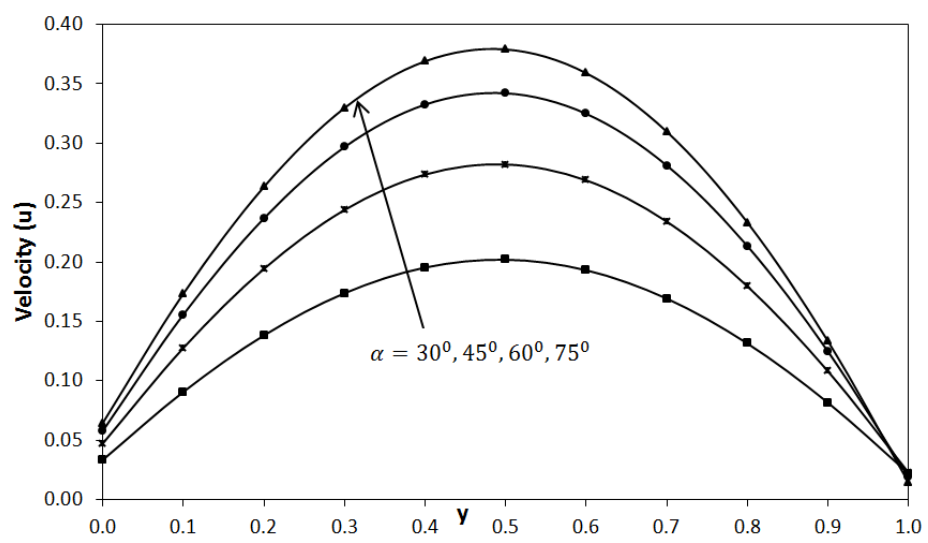

Figure 4. Effect of angle of inclination ( $\alpha$ ) on velocity profile for $\mathrm{Kn}=0.05$, $\ln =1.667, M=2.0, \eta=1, \phi=5$.

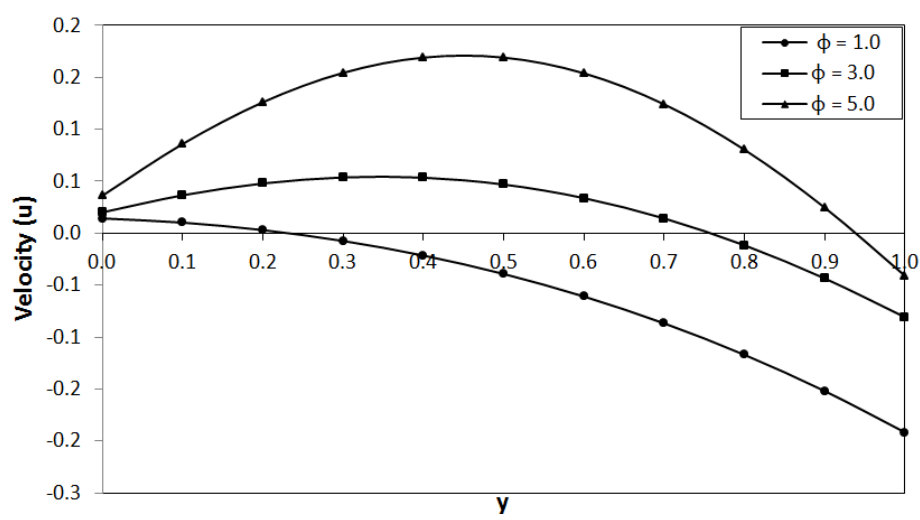

Figure 5. Effect of heat generating coefficient ( $\phi$ ) on velocity profile for $K n=0.05, \ln =1.667, M=2.0, \eta=1, \phi=5$ and $\alpha=45^{0}$.

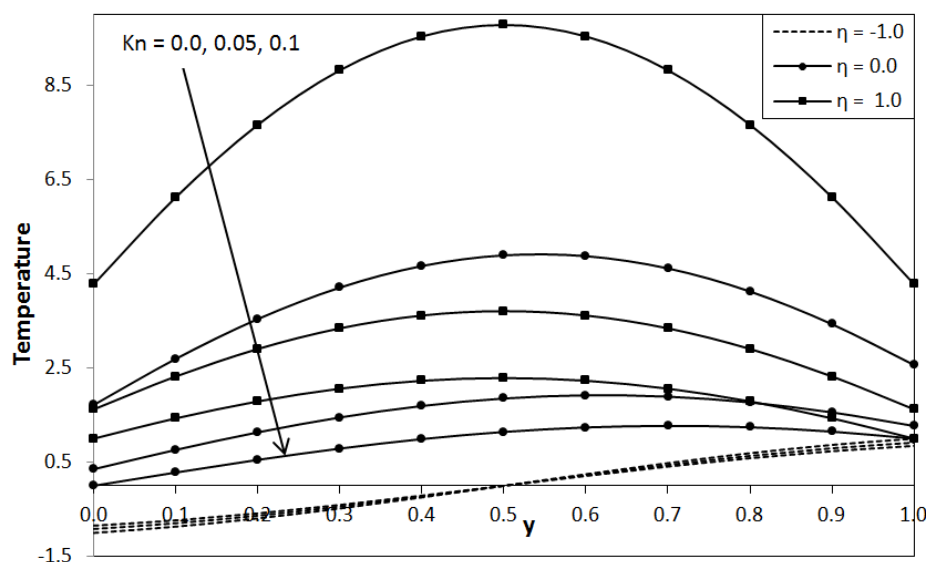

Figure 6. Effect of Kn and $\eta$ on temperature profile for $=1.667, \mathrm{Gr}=1$ and $\phi=5$.

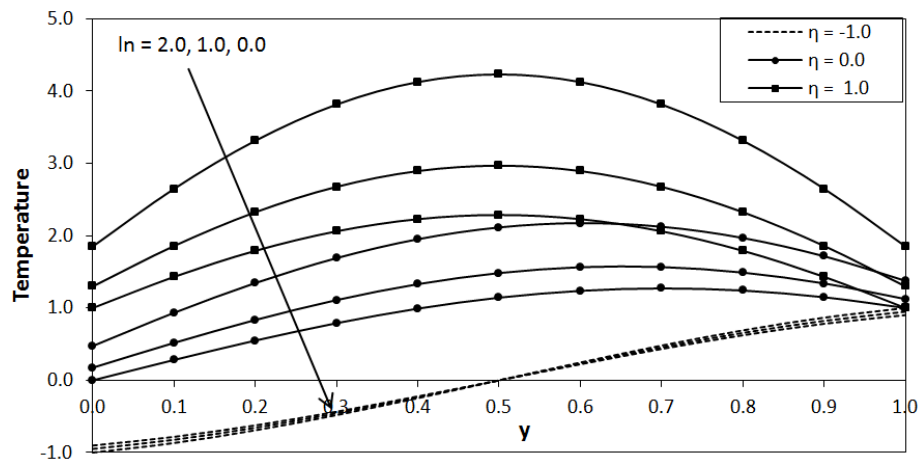

Figure 7. Effect of In and $\eta$ on temperature profile for $=0.05, \mathrm{Gr}=1, \alpha=45^{\circ}$ and $\phi=5$. 

Inclined Microchannel with Heat Generation

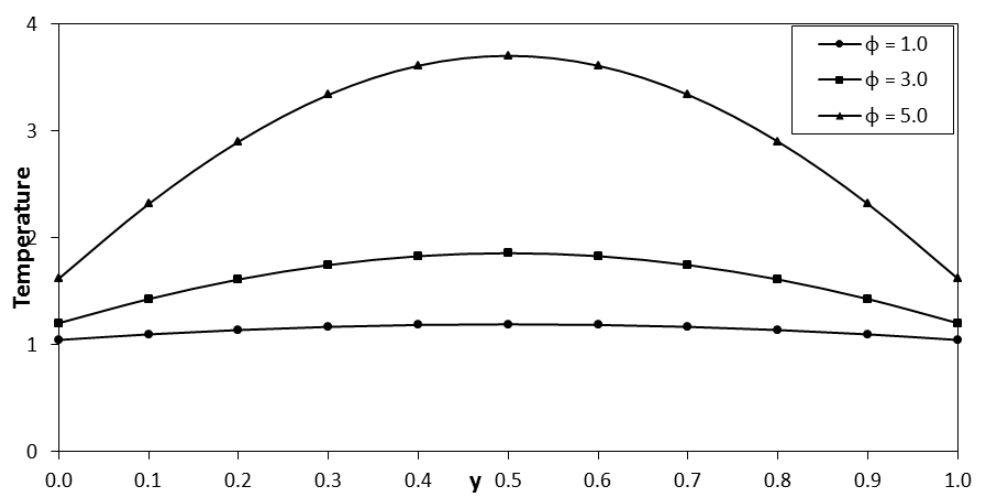

Figure 8. Effect of $\phi$ on temperature profile for $=0.05, \eta=1, G r=1$, and $\ln =1.677$.

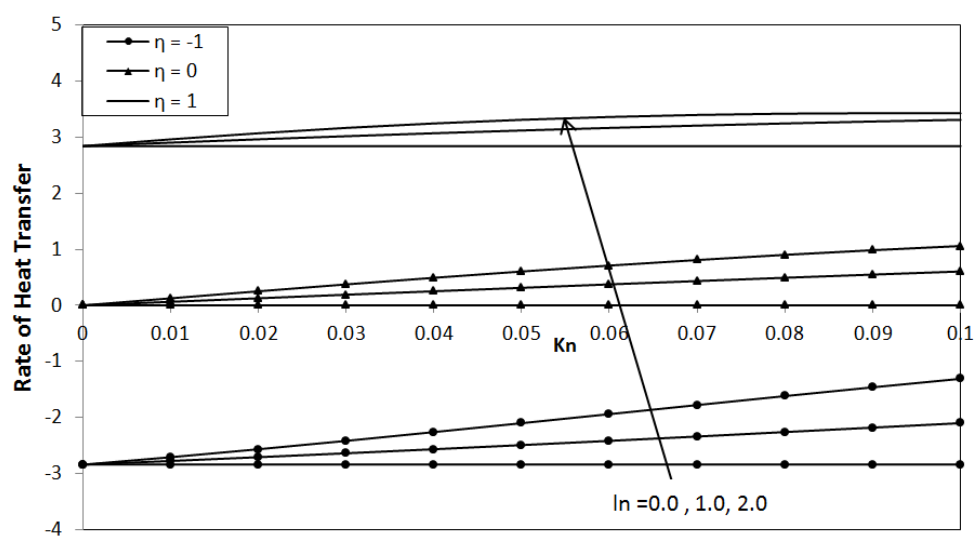

Figure 9. Rate of heat transfer (Nu1) verses Kn for the different values of $l n$.

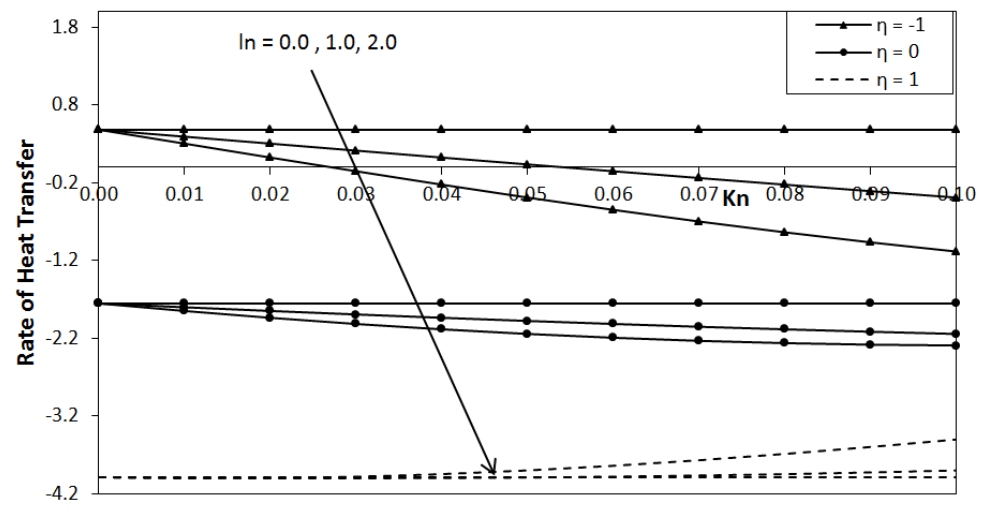

Figure 10. Rate of heat transfer (Nu2) verses Kn for the different values of $\ln$.

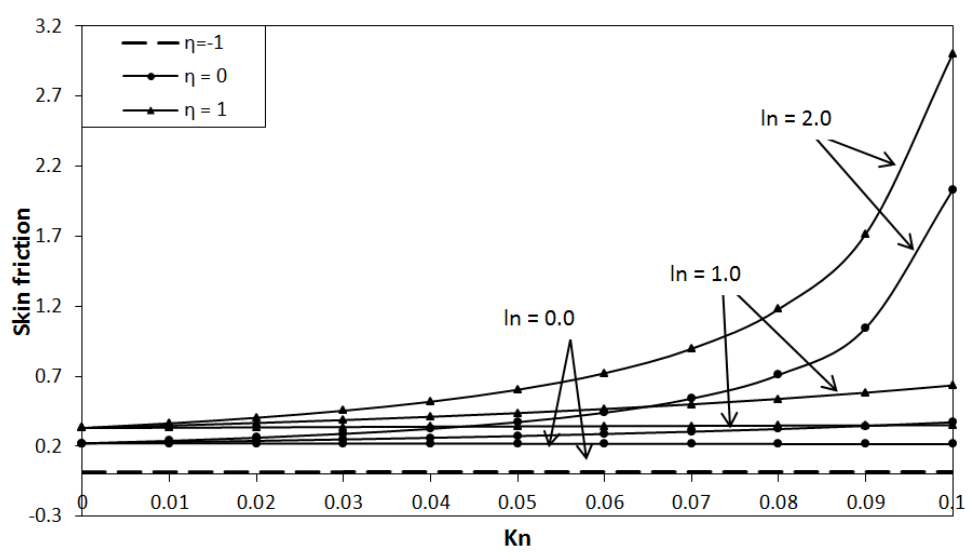

Figure 11. Skin friction $\left(\tau_{1}\right)$ for different values of $\mathrm{ln}$. 


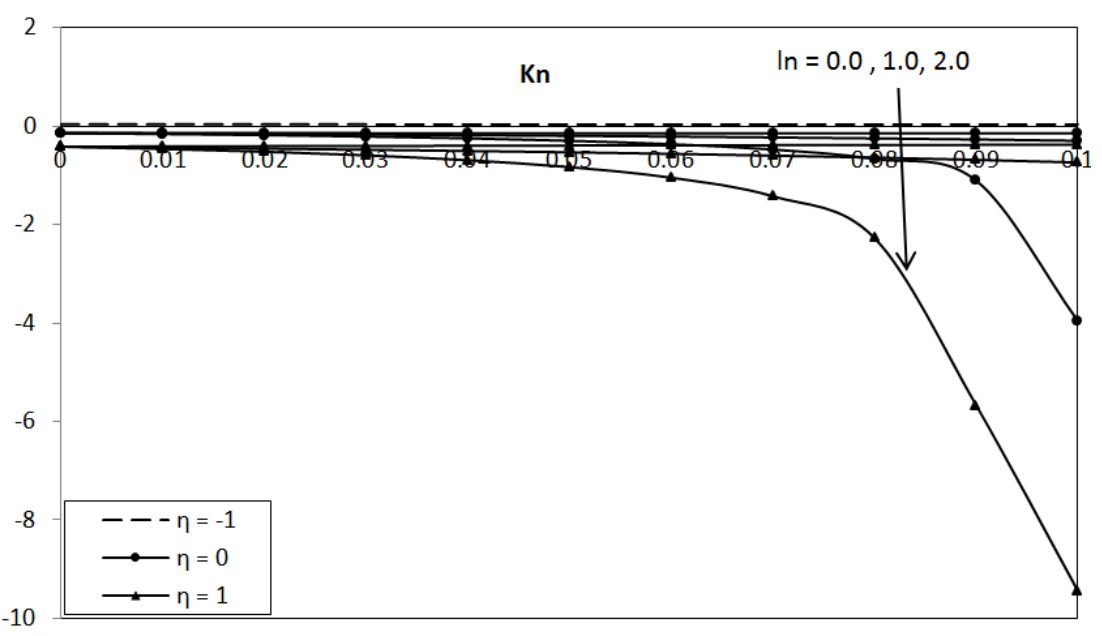

Figure 12. Skin friction $\left(\tau_{2}\right)$ for different values of $\ln$.

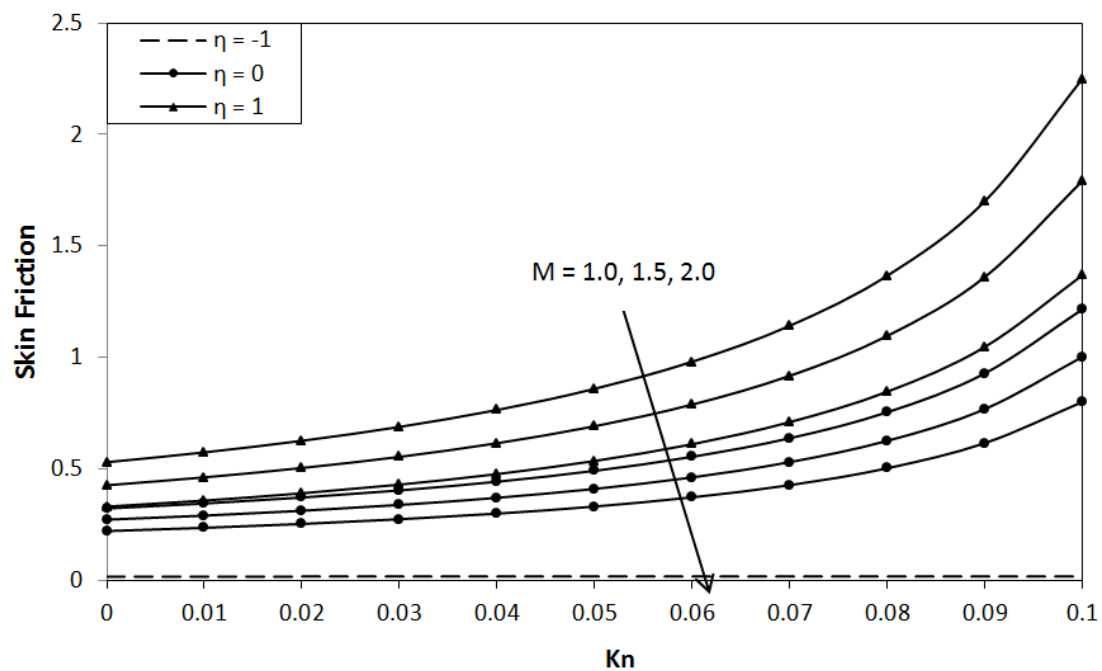

Figure 13. Skin friction $\left(\tau_{1}\right)$ for different values of $M$.

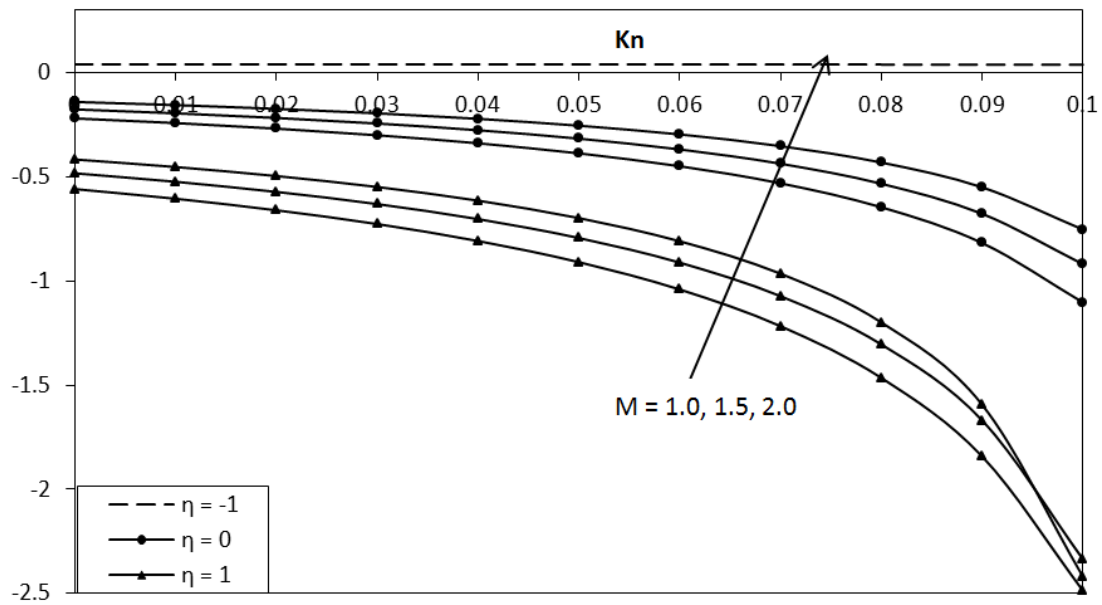

Figure 14. Skin friction $\left(\tau_{2}\right)$ for different values of $M$.

\section{Nomenclature}

$h \quad$ Width of channel

$B_{0} \quad$ Induced Magnetic field

$g$
Greek letters

$\beta$

$\alpha$
Coefficient of thermal expansion

Angle of inclination 


$\begin{array}{lll}C_{p}, C_{v} & \begin{array}{l}\text { Specific heat of fluid at constant pressure } \\ \text { and volume respectively }\end{array} & \mu \\ \ln & \text { Fluid wall interaction parameter } & \beta_{t}, \beta_{v} \\ M & \text { Hartmman Number } & \phi \\ P r & \text { Prandtl Number } & K n \\ T & \text { Fluid Temperature } & \lambda \\ T_{0} & \text { Temperature at a reference point } & \gamma_{s} \\ Q & \text { Dimensional internal heat generation or } & \sigma_{v}, \sigma_{t} \\ u_{0} & \text { absorption } & \sigma \\ u & \text { Average velocity } & \eta \\ T_{1}, T_{2} & \text { Temperature of velocity } & y=1 \text { respectively } \\ N u & \text { Nusselt number } & \tau_{1}, \tau_{2} \\ & & \end{array}$

\section{References}

[1] E. B. Akilic, M. A. Schmidt, and K. S. Breuer, "Gaseous slip flow in long microchannels". J. Microelectomech. syst. (1997), 6, 167-178.

[2] S. W. Janson, H. Helvajian and K. Breuer, "MEMS, Micorengineering and Aerospace System," In. $30^{\text {th }}$ AIAA Fluid Dyn. Conf., (1999), Norfolk, Virginia, AIAA 99-3802.

[3] M. Gad- el- Hak, "The Fluid Mechanics of Microdevices: The Freeman Scholar Lecture”. J. Fluid Eng., (1999), 121, 5-33.

[4] C. K. Chen, H. C. Weng, "Natural convection in a Vertical Microchannel." J. Heat Transfer., (2005), 127, 1053-1056.

[5] H. C. Weng, C. K. Chen, "Variable Physical Properties in natural convective gas Microflow." J. Heat transfer., (2008), 130,082401 .

[6] H. C. Weng, C. K. Chen, "On the importance of thermal Creep in natural convective gas microflow with wall heat fluxes." J. Phys. D: appl. Phys., (2008), 41, 115501.

[7] B. Buonomo, O. Manca, "Natural convection slip flow in a vertical microchannel heated at uniform heat flux." Int. J. of Thermal Sciences, (2010), 49, 1333-1334.

[8] G. Karniadakis, A. Beskok, Microflow fundamentals and Simulation. Springer Verlag, New York, (2002).

[9] Eckert ERG, Draeke Jr RM, Analysis of heat and mass transfer. Mcgraw Hill, New york, (1972),

[10] B. K. Jah, B. Aina, A. T. Ajiya, "MHD natural convection flow in a vertical parallel plates microchannel," Aim Shams Engg. Journal, (2015), 6, 289-295.

[11] A. J. Chamkha, "Unsteady Laminar Hydromagnetic FluidParticle Flow and Heat Transfer in Channels and Circular Pipes". International Journal of Heat and Fluid Flow, (2000), 21, 740-746,

[12] A. J. Chamkha, A. M. Aly, "MHD Free Convection Flow of a Nanofluid past a Vertical Plate in the Presence of Heat Generation or Absorption Effects." Chemical Engineering Communications, (2011), 198, 425-441.
Viscosity of the fluid

Dimensionless variables

Dimensionless internal heat generation coefficient

Knudsen Number

Mean free path of molecules

Specific heats ratio

Tangential and thermal momentum

accommodation coefficients respectively

Fluid electrical conductivity

Wall ambient temperature difference ratio.

Skin friction at $y=0$ and $y=1$ respectively

[13] A. J. Chamkha, A. M. Rashad, Ch. Ram Reddy and P. V. Murthy, "Effect of Suction/Injection on Free Convection along a Vertical Plate in a Nanofluid Saturated Non-Darcy Porous Medium with Internal Heat Generation." Indian Journal of Pure and Applied Mathematics, (2014), 45, 321-341.

[14] A. F. Khadrawi, A. Othman, M. A. Al- Nimr, "Transient free convection fluid flow in a vertical micro channel as described by the hyperbolic heat conduction Model," Int. j. Thermophys., (200)5, 26, 1053-1056.

[15] Hasan Nihal Zaidi, Naseem Ahmad, MHD convection flow of two immiscible fluids in an inclined channel filled with heat generation/absorption, American j. Applied Mathematics, (2016), 4, 80-91.

[16] C. C. Wang, W. J. Chang, C. H. Dai, Y. T. Lin, K. S. Yang, "Effect of inclination on the convective boiling performance of a microchannel heat sink using HEF - 7100," J. Experimental Thermal and fluid Science,( 2012), 36, 143148 ,

[17] Hartmann, J., "Hg-dynamics I Theory of the Laminar Flow of an Electrically Conductive Liquid in a Homogeneous Magnetic Field," kgl Danskevidenskab Selkab Mat Fys Medd., (1937), 15.

[18] Seigel, R., "Effect of Magnetic Field on Forced convective Heat transfer in a Parallel Plate Channel,". J Appl. Mech. (1958), 25, 415.

[19] Osterle, J. F., Young, F. J., "Natural Convection Between Heated Vertical Plates in a Horizontal Magnetic Field," J. Fluid Mech., (1961), 11, 512-518.

[20] Perlmutter, M., and Seigal, R., "Heat Transfer to an Electrically Conducting Fluid Flowing in a Channel with Transverse Magnetic Field,” NACA TN D-875, (1961).

[21] Romig, M. F., "The Influence of Electric and Magnetic Fields on Heat Transfer to Electrically Conducting Fluids," Adv. Heat Transfer, (1961), 1, New York, Academic Press,

[22] Umavathi, J. C., "A Note on Magnetoconvection in a Vertical Enclosure,” Int. J. Nonlinear Machanics, (1996), 31, 371-376.

[23] Naseem Ahmad, Hasan Nihal Zaidi, "Magnetic effect on unsteady oberbeck convection through vertical stratum," Int. j. heat and Technology, (2006), 25, 12-131. 